\title{
Challenges in Decision Making in Elderly Patients with Gastrointestinal Malignancies
}

\author{
Chair: \\ Ulrich Wedding a \\ Participants: Riccardo A. Audisio ${ }^{b}$ Konrad Klaus Richter ${ }^{c, d}$ Siri Rostoft ${ }^{e}$ f \\ a Department of Internal Medicine II (Haematology, Oncology, Palliative Care), University Hospital Jena, Jena, Germany; \\ ${ }^{b}$ Department of Surgery, University of Liverpool, St Helens Teaching Hospital, St Helens, UK; \\ 'Department of Surgery, Southland Hospital, Invercargill, New Zealand; \\ ${ }^{\mathrm{d}}$ Dunedin Hospital and School of Medicine, Dunedin, New Zealand; \\ e Department of Geriatric Medicine, Oslo University Hospital, Oslo, Norway; \\ f University of Oslo, Oslo, Norway
}

\begin{abstract}
Question 1: In your institution, do you routinely perform a geriatric assessment (GA) in older adults with cancer of the gastrointestinal tract? If yes, how did you succeed to do so; if not, what are the barriers?
\end{abstract}

Audisio: It has been a decade since my interest has focused mainly on breast cancer, where I use GA occasionally: after checking for cognitive impairment I perform a structured GA rather selectively.

In the gastrointestinal environment the routine use of GA (or screening tools in its place), although mandatory, has not been taking place - yet. Physicians are very conservative and reluctant to think out of the box. Protocols are rigid and obsolete: they include a number of expensive tests, but tend to forget inexpensive processes which are known to associate to better outcomes, shorter hospital stay, and reduced costs. The presence of an onco-geriatric nurse would be highly helpful.

Richter: Yes, we are routinely performing basic GAs during the surgical preadmission clinic. Also, an additional anesthetic preadmission clinic is run by the anesthetist. Their input in the decision making whether a patient is fit for cancer surgery or not is crucial. We are planning on introducing 'Timed Up and Go' (TUG), Activities of Daily Living (ADL) as well as Instrumental Activities of Daily Living (IADL), and Comprehensive Geriatric Assessment (CGA).

Rostoft: No, we do not. The first barrier would be to get the physicians involved in the care of older patients with cancer to realize the need for a GA or at least a frailty screening. We have been able to implement frailty screening in other departments, e.g. the cardiology department. Secondly, there must be a system in place to deal with the results from the frailty screening or the GA. I think it is difficult to implement new forms to fill in unless there is a clear clinical benefit from the start.

Question 2: Pre- instead of re-habilitation might be a good strategy to improve the preoperative condition of the patient and to have a better outcome. What are the major pros and cons according to your opinion?

Audisio: Nutritional impairment is highly prevalent amongst older patients, affecting one in two of them. Postoperative complications are likely to occur in malnourished patients, even if the malnutrition is only mild. Cancer surgery is rarely performed as an emergency; therefore, surgical oncologists have all the time to reconfigure their patients: correcting anemia and hydroelectrolytic balance, fostering motivation and mental wellness, performing active physiotherapy and cardiopulmonary exercise tests (CPET).

A number of recommendations are to be considered postoperatively, as per the well-known ERAS (Enhanced Recovery After Surgery) study group. Despite all the evidence they have put together to support enhanced recovery protocols, yet again patients (especially elderly surgical patients) are mobilized too late, catheters are not removed, oral feeding is postponed, and discharge from the intensive care unit is delayed. These are simple and cheap measures which should be taken into consideration as a matter of priority.

Richter: Pre-habilitation has become part of our ERAS protocol. A colorectal nurse specialist is championing pre-habilitation. In our team, we make sure that our ERAS standard protocol is well known and correctly followed. The newly introduced 'pre-habilita-

\section{KARGER}

(c) 2017 S. Karger GmbH, Freiburg

Fax +497614520714
PD Dr. Ulrich Wedding

Abteilung Palliativmedizin, Klinik für Innere Medizin II

Universitätsklinikum Jena

Bachstraße 18, 07743 Jena, Germany

Ulrich.Wedding @ med.uni-jena.de 
tion' requires the education of other specialties, including nursing, anesthesia, and physiotherapy. To us, pre-habilitation only offers benefits, i.e. better physical and mental fitness of the patient in preparation for a planned surgical procedure.

Rostoft: Firstly, I believe that it is not 'either or' - both pre-habilitation and re-habilitation are necessary. Pros for pre-habilitation are optimization of patients in order to reduce complications and active involvement of patients in their own care. If the pre-habilitation is home-based, another advantage would be to establish contact between the patient and the community services before active treatment is started - I believe this could improve the treatment trajectory. Cons are costs, organizational issues, and if the pre-habilitation is in-hospital: logistical challenges/time investment for the patients. In theory, if pre-habilitation reduces the rate of complications, it could lead to more efficient re-habilitation as well. I believe in maintaining a focus on sustaining functional status all the way through the treatment trajectory. Both pre-habilitation and re-habilitation fit well into this line of thinking. The key goal would be to minimize functional decline and to improve quality of life during and after treatment in patients with gastrointestinal cancer.

\section{Question 3: Postoperative delirium is a major side effect of anesthesia and surgery with short- and long-term negative outcome. What are the strategies at your institution to detect patients at risk and to avoid the occurrence?}

Audisio: I agree that we do not do enough to prevent and detect postoperative delirium early. Having said that, we have a nurse-led questionnaire which is repeated daily after surgery.

Awareness among the treating team is pivotal: routinely use of restraints, catheterization, polypharmacy, metabolic disorders, dehydration, infection, confrontations/arguments, and moving from ward to ward are risk factors; recognizing lack of concentration, reduced physical function, falls, poor appetite, insomnia, and hallucinations might lead to early detection of postoperative delirium.

Richter: Our anesthetists try to avoid long-acting opioids during and after the surgical procedure. This, however, requires repeated discussion and education of new and locum members of the team.

Rostoft: To detect risk: Pre-treatment cognitive assessment. Patients with cognitive impairment have a much higher risk of delirium. Strategies to avoid delirium: Removal of anticholinergic drugs, avoid transferring a patient between wards, establish normal day/night routine, use caregivers to reduce stress of new environment, avoid unnecessary sedatives, treat underlying conditions early (infection, anemia, hypoxia, pain etc.) - maintaining homeostasis. Make sure glasses and hearing aids are accessible. Train all staff in recognizing and handling delirium - it is recommended to check patients actively, e.g. by using the 4 'A's Test (4AT), but we have not implemented this in our institution. If delirium develops
- always look for the underlying medical reason and treat that. I also think that it may help patients and caregivers to inform about an increased risk of delirium if the patient has cognitive impairment. Delirium is reported as a frightful experience both for the patient and the caregivers, and maybe talking about the risk upfront could relieve some of that stress.

\section{Question 4: New anticancer drugs are often very expensive. Are the prize of a drug and the age of a patient criteria for or against treatment? Should they be a criterion?}

Audisio: The medical community, with the assistance of patient representatives, should start questioning oncological achievements right now: the 91 new therapies approved for solid tumors between 2002 and 2016 have an overall survival gain of 2.2 months; most of them cost over USD 100,000/year. We have been offered a severe misrepresentation of modern oncology. This is indeed a very crucial problem and not only an issue for older patients.

Richter: As a surgeon, I am not intensively involved in the decision making whether a chemotherapy treatment should be offered. I am, however, a member of our multidisciplinary management (MDM) tumor board. As surgeons we have often diagnosed our patients first and dealt with them before the surgery. Therefore, we seem to know them well and perhaps better than medical or radiation oncologists. From my point of view, we rarely have a discussion about the financial implications should a new anticancer drug be offered. Discussion points should include life expectancy as well as the patient's preference.

In our financially restricted tax money-funded Australasian health system, the use of new expensive anticancer drugs should be carefully assessed and balanced against perhaps other usage options, i.e. buying medical or surgical equipment and hiring specialists and psychologists. This discussion is, unfortunately, not happening yet enough.

Rostoft: In Norway, age is not used as a criterion per se. Norway is an egalitarian society. In general, it does not make sense to use age as a sole criterion for treatment decisions in my opinion since the health status and life expectancy of a patient depend more on frailty, comorbidities, and disability.

\section{Question 5: Some current data support the early integration of palliative care into oncologic treatment. Which approach do you pursue at your institution and how would you recommend to involve palliative care?}

Audisio: My practice as a surgical oncologist does not expose me to terminal cases which are usually handled between medical/ clinical oncologists and palliative care specialists. 
Richter: We have a new 10-bed hospice which is affiliated with the hospital. Right now it is run by an American qualified palliative physician. The early involvement of palliative care and hospice is happening daily, but it apparently also depends on the surgeon's communication skills. If the surgeon only has a limited understanding of what palliative care and hospice can do for their patients, then the involvement of the palliative care team will be delayed.

Rostoft: This varies between institutions in Norway. In my institution, we have good access to the palliative care team for in-hospital patients with cancer. This is necessary and useful in everyday clinical practice. I do not work in the cancer department, but in the geriatric department we have a low threshold to refer to the palliative care team.

\section{Question 6: When looking into the future, what are} the major improvements in the medical treatment of older adults with cancer of the gastrointestinal tract you would like to achieve in the next decade?

Audisio: Whatever the patient's age, surgery is the least expensive and most curative treatment. Thus, there is no doubt that major improvements should focus on optimizing surgical practice. We are eagerly looking at the findings of the GOSAFE study which will point out the selection criteria and perioperative steps to set in place in view of optimizing the care of patients with cancer of the gastrointestinal tract.

Richter: Hopefully, we will have better and more potent drugs available in the future that have fewer side effects and are financially sustainable. For a surgeon, major improvements will come through more advanced minimally invasive surgery, better imag-

\section{Participants}

Riccardo A. Audisio, MD, FRCS, PhD (Hon)

Department of Surgery, University of Liverpool

St Helens Teaching Hospital

Marshalls Cross Road, St Helens, WA9 3DA, UK

raudisio@doctors.org.uk

Assoc. Prof. Konrad Klaus Richter, MD PhD (PD Dr. med. habil.), FRACS

Department of Surgery, Southland Hospital

Kew Road, Invercargill 9810, New Zealand

konradklaus.richter@gmail.com ing, and early detection and treatment. In the future, more focus should be put on prevention, prophylaxis, and early diagnosis. In New Zealand, according to the PIPER study, almost $30 \%$ of patients with colorectal cancer present late or acutely in the emergency department. To improve this situation, we currently do not need more chemotherapy drugs or surgical equipment but rather a better public self-awareness and the avoidance of risk factors, i.e. alcohol, non-healthy diet, and inactivity. Governments should fund screening, colonoscopies, and a health workforce that communicates these problems with the public.

Rostoft: My visions for the future are: implementation of frailty screening aiming to identify patients with high risk of complications and patients with cognitive impairment before starting the treatment trajectory. I think that it is necessary to improve the selection of patients for various treatments to avoid undertreatment and overtreatment, and that GA is the best way to do this. A GA also provides an essential baseline before starting treatment. Without a baseline it is difficult to detect e.g. functional decline. It is also a major issue to bridge the gap between hospital care and home care by improving the collaboration. Patients and caregivers report that specialists and home care services often do not communicate, and as a consequence the patients find it difficult to get help when they need it because it is unclear who has the responsibility. Older patients with cancer often have comorbidities, use several drugs, and may have functional and cognitive limitations. They need their physicians to look at their overall goals, and help them navigate treatment and drugs in the most optimal way to reach those goals. This requires a holistic approach to the patient, smooth communication between specialist and community care, and the investment of time before treatment is started to assess the patient, to establish the individual risk of treatment complications, and to talk about the patient's preferences.
Siri Rostoft, MD, PhD

Department of Geriatric Medicine

Oslo University Hospital

$\mathrm{Pb} 4956$ Nydalen, 0424 Oslo, Norway

srostoft@gmail.com 